\title{
Stable Gastric Pentadecapeptide BPC 157, Robert's Stomach Cytoprotection/Adaptive Cytoprotection/Organoprotection, and Selye's Stress Coping Response: Progress, Achievements, and the Future
}

\author{
Predrag Sikiric ${ }^{1}$, Ki-Baik Hahm ${ }^{2}$, Alenka Boban Blagaic ${ }^{1}$, Ante Tvrdeic ${ }^{1}$, Katarina Horvat Pavlov ${ }^{3}$, Andrea Petrovic ${ }^{3}$, Antonio \\ Kokot $^{1}$, Slaven Gojkovic ${ }^{1}$, Ivan Krezic ${ }^{1}$, Domagoj Drmic ${ }^{1}$, Rudolf Rucman ${ }^{1}$, and Sven Seiwerth ${ }^{3}$ \\ ${ }^{1}$ Department of Pharmacology, Medical Faculty, University of Zagreb, Zagreb, Croatia, ${ }^{2}$ Digestive Disease Center, CHA Bundang Medical Center, \\ CHA University School of Medicine, Seongnam, Korea, and ${ }^{3}$ Department of Pathology, Medical Faculty, University of Zagreb, Zagreb, Croatia
}

We reviewed again the significance of the stable gastric pentadecapeptide BPC 157 as a likely mediator of Robert's stomach cytoprotection/adaptive cytoprotection and organoprotection and as novel mediator of Selye's stress coping response to reestablish homeostasis. Specific points of BPC 157 therapy and the original concept of Robert's cytoprotection/adaptive cytoprotection/organoprotection are discussed, including the beneficial effects of BPC 157. First, BPC 157 protects stomach cells and maintains gastric integrity against various noxious agents (Robert's killing cell by contact) and is continuously present in the gastric mucosa and gastric juice. Additionally, BPC 157 protects against the adverse effects of alcohol and nonsteroidal anti-inflammatory drugs on the gastric epithelium and other epithelia, that is, skin, liver, pancreas, heart (organoprotection), and brain, thereby suggesting its use in wound healing. Additionally, BPC 157 counteracts gastric endothelial injury that precedes and induces damage to the gastric epithelium and generalizes "gastric endothelial protection" to protection of the endothelium of other vessels (thrombosis, prolonged bleeding, and thrombocytopenia). BPC 157 also has an effect on blood vessels, resulting in vessel recruitment that circumvents vessel occlusion and the development of additional shunting and rapid bypass loops to rapidly reestablish the integrity of blood flow (ischemic/ reperfusion colitis, duodenal lesions, cecal perforation, and inferior vena caval occlusion). Lastly, BPC 157 counteracts tumor cachexia, muscle wasting, and increases in pro-inflammatory/procachectic cytokines, such as interleukin- 6 and tumor necrosis factor- $\alpha$, and significantly corrects deranged muscle proliferation and myogenesis through changes in the expression of FoxO3a, p-AKT, p-mTOR, and p-GSK-3 $\beta$ (mitigating cancer cachexia). (Gut Liver 2020;14:153-167)

Key Words: Stable gastric pentadecapeptide BPC 157 therapy; Robert's cytoprotection; Adaptive cytoprotection; Organoprotection concept; Mediator

\section{INTRODUCTION}

Stable gastric pentadecapeptide BPC 157 is a novel anti-ulcer peptide, used in trials for the treatment of ulcerative colitis and now multiple sclerosis, lethal dose (dosage required to kill 1\% of the test population, LD1) not reported. ${ }^{1-13}$ We recently reviewed its significance ${ }^{1-13}$ as a likely mediator of the Robert's stomach cytoprotection/adaptive cytoprotection ${ }^{14-19}$ and organoprotection, ${ }^{20,21}$ as well as a novel mediator of the Selye's stress coping response, ${ }^{22-25}$ generally known, but still not completely clarified.

Briefly, to support the pleiotropic beneficial effects of BPC 157 application, ${ }^{1-13}$ we will review the particular background of these essential holistic concepts, the Robert's stomach cytoprotection $^{14-19}$ and organoprotection ${ }^{20,21}$ and the Selye's stress coping response (Fig. 1). ${ }^{22-25}$

Background of the Robert's concept (stomach cytoprotection and organoprotection) versus the Selye's concept (stress coping response)

Robert's stomach cytoprotection ${ }^{14-19}$ and organoprotection ${ }^{20,21}$ and the Selye's stress coping response $\mathrm{e}^{22-25}$ both intend to reestablish homeostasis. In the late 1970's the concept of Robert's cytoprotective homeostasis (cell protection) was related to the

Correspondence to: Predrag Sikiric

Department of Pharmacology, Medical Faculty, University of Zagreb, Salata 11, POB 916, Zagreb 10000, Croatia

Tel: +385-1-4566-833, Fax: +385-1-4811-244, E-mail: sikiric@mef.hr

Received on November 5, 2018. Revised on January 10, 2019. Accepted on January 21, 2019. Published online June 4, 2019. pISSN 1976-2283 eISSN 2005-1212 https://doi.org/10.5009/gnl18490

@ This is an Open Access article distributed under the terms of the Creative Commons Attribution Non-Commercial License (http://creativecommons.org/licenses/by-nc/4.0) which permits unrestricted non-commercial use, distribution, and reproduction in any medium, provided the original work is properly cited. 
Stable gastric pentadecapeptide BPC 157 and implementation of the Robert's stomach cytoprotection/organoprotection concept

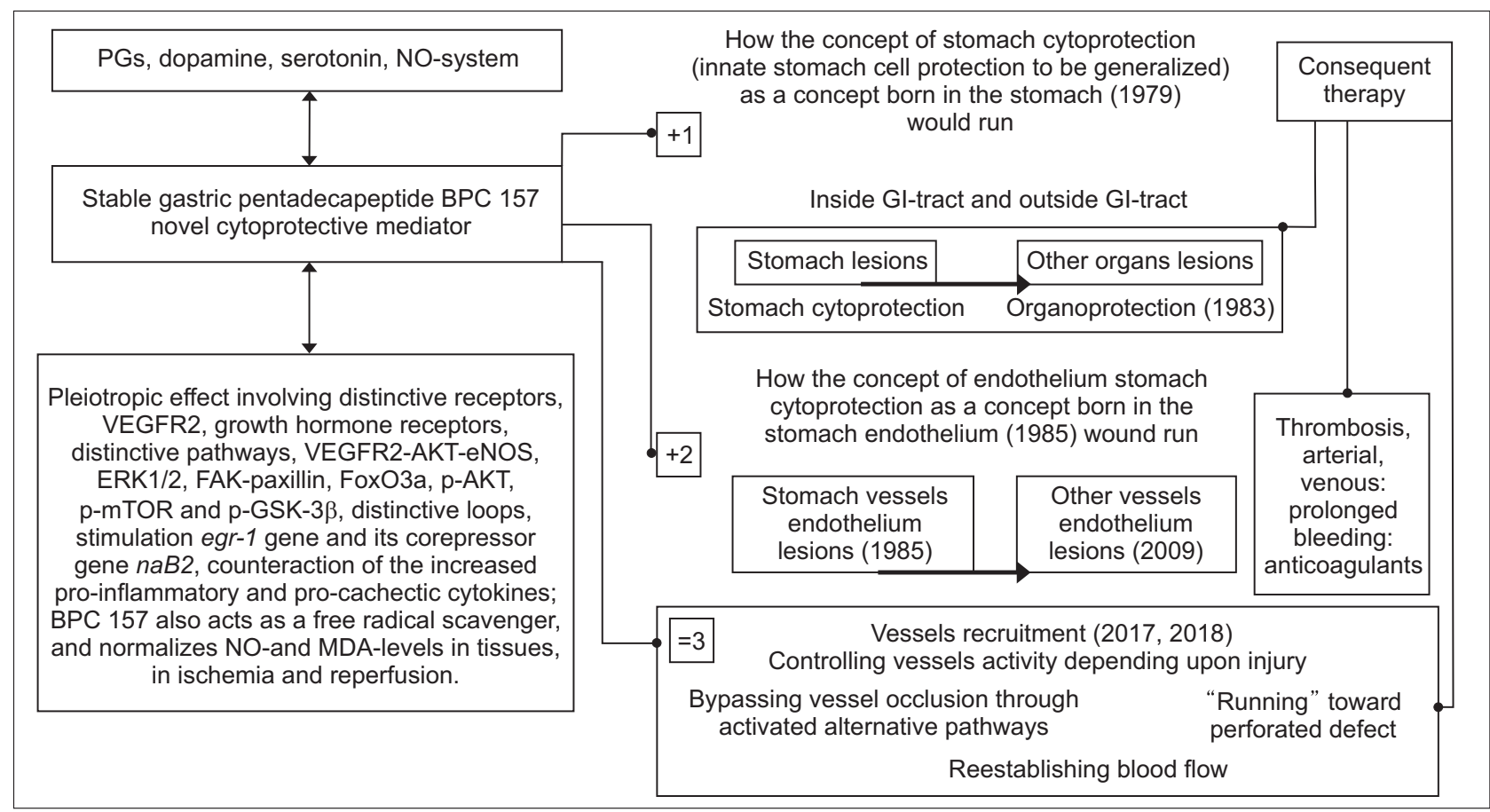

Fig. 1. BPC 157 has beneficial effects and thus leads to stomach cytoprotection $\rightarrow$ organoprotection of the whole gastrointestinal tract, including both prophylactic and therapeutic effects for pre-existing lesions in individuals with the most complex disturbances, such as internal and external fistulas, or anastomosis complicated with severe colitis (indicated as +1$){ }^{1-13}$ In addition, there is a particular effect on endothelial integrity (indicated as +2$)$. $^{1-13}$ Together, these may result in the particular activation of blood vessels during injury, vessel occlusion, or organ perforation, the recruitment of the vessel to organize an adequate shunting and bypass occlusion (indicated as=3). ${ }^{101,113,117,118}$ Furthermore, the effect of BPC 157 is due to its interaction with and modulation of the NO system, and its interaction with prostaglandin, dopamine, and serotonin systems has also been documented. ${ }^{1-13}$ BPC 157 also acts as a free radical scavenger, counteracts free radical-induced lesions, and normalizes NO and MDA levels in tissues and during ischemia and reperfusion. ${ }^{101,113,114,116-118}$ Subsequent studies from other groups ${ }^{2,96-101}$ have confirmed our original findings. ${ }^{65-78}$ Pleotropic effects involving distinctive receptors, including VEGFR2 and growth hormone receptors, distinctive pathways, including VEGFR2-AKT-eNOS, ERK 1/2, FAK-paxillin, FoxO3a, p-AKT, pmTOR and p-GSK-3ß, and distinctive loops, including stimulation of the egr-1 gene and its corepressor gene naB2, and counteraction of increases in pro-inflammatory and procachectic cytokines, ${ }^{2,96-101}$ likely minimize the inherent lack of full understanding of the mechanisms that may be involved. However, more important is the practical evidence from a considerable number of the studies, particularly in gastrointestinal research, that intragastric administration or per os administration in drinking water, is equally effective as injections of the supplement administered to rodents, which has been performed in the majority of studies on BPC $157 .{ }^{12,41,43,48,51,54,55,58,59,61-63,82-85,89,91-93,98,114,116,117,143-147,149-155}$ In reality, in particular along with its safety profile, LD-1 is not achieved, and there are no reported adverse effects in clinical trials; ${ }^{1-13}$ this evidence suggests the ease of practical clinical application. PGs, prostaglandins; NO, nitric oxide; VEGF, vascular endothelial growth factor; VEGFR2, VEGF receptor 2; eNOS, endothelial nitric oxide synthase; FAK, focal adhesion kinase; Fox03a, transcription factor; p-AKT, phospho-AKT; p-mTOR, phospho mammalian target of rapamycin; p-GSK-3 $\beta$, phospho glycogen synthase kinase $3 \beta$; MDA, malondialdehyde; GI, gastrointestinal.

stomach, ${ }^{14-19}$ but soon it was further extended (i.e., as a principle acting in the stomach mucosa integrity maintenance much like in the maintaining of the other epithelia integrity, stomach cytoprotection $\rightarrow$ organoprotection). ${ }^{20,21}$ Much earlier, Selye's concept was born outside of the gastrointestinal tract, in the adrenal gland, and Selye conceptualized the general syndrome of adaptation (i.e., adrenal gland hypertrophy, thymolymphatic involution, and stomach stress ulcers) to achieve general homeostasis in the whole body while the derangement could explain the genesis of many stress-induced diseases. ${ }^{22-25}$

\section{Hartmann's discovery of the cortin for the Selye's concept}

Of note, Selye's concept was preceded by Hartmann's discovery of cortin. ${ }^{26,27}$ Cortin was extract from the adrenal gland cortex, and called life-maintaining factor. ${ }^{26,27}$ It kept adrenal- ectomized animals alive, and was essential for the treatment of Addison disease patients. ${ }^{26,27}$ On the one hand, these outbreaks ${ }^{26,27}$ initiated the search for and discovery of corticosteroids by Kendal and Reichstein groups. ${ }^{28,29}$ On the other hand, this lead Selye to propose the concept that the most essential role of the "adrenotropic" principle in the coping response was to reestablish homeostasis ${ }^{22}$ while two other principles, "thyrotropic" and "histaminergic," remained less emphasized. ${ }^{22}$

\section{Homeostasis}

In principle, these concepts ${ }^{14-25}$ consider "homeostasis," (cytoprotection/adaptive cytoprotection, organoprotection) as the organ's protection from injury as well as organ's recovery after injury, as the most important result. The beneficial effects (endogenous and exogenous) of the agent supposed to mimic the 
activity of the supposed mediator (i.e., prostaglandins, corticosteroids) should link theory and practice. The achieved beneficial effects should be the practical realization of the theoretical point of homeostasis (cytoprotection/adaptive cytoprotection, organoprotection) in the body's maintenance of integrity. ${ }^{4}$ Unfortunately, the general but insurmountable problem is that neither of the supposed mediators when applied as a therapy could realize in practice the full theoretical assumption that "homeostasis" could be achieved. ${ }^{4}$

\section{Inadequate standard mediators activity to homeostasis in Robert's stomach cytoprotection and organoprotection and the Selye's stress coping response}

In general, the inadequate functioning of the highly sophisticated theory in real practice is due to considerable limitation in these mediators activity.

\section{Robert's stomach cytoprotection and organoprotection}

As emphasized, ${ }^{1-13}$ the beneficial effect of prostaglandins as the concept of cytoprotection only works when they are given before injury, but not when given after injury. ${ }^{14-19}$ Likewise, commonly thought to have essential wound healing potential, ${ }^{30}$ the standard peptidergic growth factors for the "wound healing concept" confront with an alike problem in practical realization of the high theoretical potential. ${ }^{1}$ The angiogenic growth factors for healing in the gastrointestinal tract, and particularly for the healing of the extra-gastrointestinal tissues (i.e., skin, tendon, ligament, muscle, and bone), regularly need special and various delivery systems and various carrier addition. ${ }^{1}$ This obstacle obscures the inherent beneficial effect of any peptide ${ }^{1}$ (making uncertain whether the peptide, or the carrier or the peptide+carrier(s) complex might be essential for the gotten effect).

\section{Selye's stress coping response}

The application of the Selye's stress coping response concept ${ }^{22}$ to resolving the practical problem of arthritis, ${ }^{31}$ resulted in the corticosteroid clinical outbreak. ${ }^{32}$ However, the first integrative factor in Selye's stress adaptive response of the body, which could have a large range of beneficial effects that could be accordingly applied as the therapy, remains largely undiscovered. ${ }^{4}$ The consequence is the evident gap between the theory (reestablished homeostasis, integrative beneficial effects) and the practical realization (limited beneficial activity of the given agent with the harmful effect as well). This missing point illustrates the misleading evidence that the foreseen integrative mediator that integrates the adaptive bodily response to stress became an argument used in favor of corticotropin releasing factor (CRF). ${ }^{33-36}$ Namely, CRF has a mild ulcerogenic effect when given centrally. ${ }^{37}$ Furthermore, even if CRF application protects against gastric ulcers, ${ }^{38}$ exogenous administration of CRF can reproduce cardinal features of diarrhea predominant irritable bowel syndrome including anxiogenic behavior, enhanced viscera pain to colorectal distention, increased colonic mucus secretion, propulsive motility, development of watery stool/diarrhea, and increased colonic mucosal permeability facilitating bacteria translocation into colonic tissue..$^{35,36}$ Thus, a negative syndrome is quite far from the integrative beneficial effect ascribed to the putative Selye's first integrative mediator. ${ }^{22-25}$ Likewise, unlike the secretion of adrenocorticotropic hormone, adrenalin and corticosteroids which are released into the bloodstream to meet the threat, these integrative beneficial effects could not be achieved with the administration of corticosteroids. ${ }^{4}$ This should not be the case in the gastrointestinal tract where corticosteroids induce gastric lesions while prednisolone induced intestinal lesions (which were characteristic injuries) serve to establish prostaglandins cytoprotection in the intestine. ${ }^{14-21}$ Consequently, as we emphasized, ${ }^{4}$ since the original organoprotective/healing intention of stress coping response ${ }^{22}$ confronts with unsuccessful realization by the Selye's "first mediator" in stress response, that integrative mediator that integrates the adaptive bodily response to stress, remains obscured in the highly sophisticated latter Selye's stress concept presentation. ${ }^{25}$

A point of disillusionment appeared in early 1980's with the notion that Selye's concept should be replaced with humbler but more precise ideas. ${ }^{39}$ An increase in hypothalamo-pituitary adrenal activity is seen as having adaptive endocrine effects and behavioral consequences via its influence on memory and arousal; and economy of the body from the way peptides play different function depending upon their localization in the body. ${ }^{39}$

\section{Link between Robert's concept and Selye's stress coping} response concept

Thereby, taking these general caveats into account, an interesting link between Robert's concept ${ }^{14-21}$ and Selye's stress coping response concept, ${ }^{22-25,40}$ recently noticed, ${ }^{4}$ is that Robert, with his prostaglandins stomach cytoprotection/adaptative cytoprotection/organoprotection concept, ${ }^{14-21}$ actually accommodates and extends Selye's stress concept ${ }^{22,40}$ toward the stomach and gastrointestinal tract. Robert's stomach cell protection (cytoprotection) appears as Selye's "response to damage as such."22 Robert's small irritant that protects against strong irritant (adaptative cytoprotection $)^{17}$ corresponds to Selye's small stress that protects against severe stress (general adaptation). ${ }^{40}$ Robert's stomach cell protection (cytoprotection) ${ }^{14-19}$ extended to other organs (i.e. liver, pancreas) (organoprotection) ${ }^{20,21}$ appears as Selye's reestablished homeostasis ${ }^{22}$ (as an organoprotective stress coping response that should eliminate damage in any organ (organoprotection), readily reproduced by such agents application). ${ }^{22}$ Robert's nonspecific necrotizing agents (strong alcohol, strong base, hypertonic solution, boiled water, given intragastrically; nonsteroidal anti-inflammatory drugs [NSAIDs] and prednisolone given systemically), severe necrotizing stomach lesions 
and perforating intestinal lesions ${ }^{14-19}$ corresponded to the Selye's diverse nocuous agents given systemically and left the organism severely damaged. ${ }^{4}$

Thus, as we already emphasized, ${ }^{4}$ it follows that these two concepts have very close interconnections, and thereby, both merit common solution.

\section{Significance of the pleotropic beneficial effects for Robert's concept (stomach cytoprotection/adaptative cytopro- tection $\rightarrow$ organoprotection) and Selye's stress coping response concept}

Of note, Robert's concept (stomach cytoprotection/adaptative cytoprotection $\rightarrow$ organoprotection) on which more focus is put on may be more workable. However, "homeostasis" (i.e., in the stomach as stomach cytoprotection/adaptative cytoprotection, and in the other organs as organoprotection) still warrants eventual realization in more advanced practice (providing that prostaglandins analogues have no more than marginal application in gastroduodenal lesions therapy). ${ }^{11}$

The antecedent Selye's organoprotective issue, "the damage" consequent to acute nonspecific nocuous agents that produce "the organism severely damaged" in rat experiments, ${ }^{22}$ since the very beginning, clearly indicated what should be the essential proof of concept and interposed agent activity supposed to be involved in the counteractive coping response organization. Evidently, the antecedent cortin was the prototype with its activity (and its huge range of beneficial effects). ${ }^{26,27}$ Thereby, this proof has to be a pleotropic beneficial effect as the end result of the application of agents that were supposed to have the essential integrative role of the Selye's (still putative) mediator that integrates the adaptive bodily response to stress ${ }^{22,25}$ whatever particular mechanism lies behind it. ${ }^{22,25}$

Likewise, the resolving of the Robert's stomach cytoprotection/adaptative cytoprotection concept and its extension to the other organs lesions (cell protection that should be generalized) would occur by application of the suited agents capable to achieve such large range of the beneficial effect (stomach cytoprotection $\rightarrow$ organoprotection=Selye's homeostasis). At the same time, this contention realized (demonstrable pleotropic beneficial effects with the given agent) could mean the practical realization of the theory and accommodations of the previous Selye's original stress concept ${ }^{22}$ and subsidiary concepts, braingut concepts, brain peptides, angiogenic growth factors activities. $^{4}$

This contention brings us to the elaborate again the significance of the pleotropic beneficial effects of the BPC 157 application along with elaboration of the original Robert's cytoprotection/adaptive cytoprotection/organoprotection concept.
Original Robert's cytoprotection/adaptive cytoprotection/ organoprotection concept

Elaboration with the BPC 157 versus elaboration with prostaglandins, somatostatin, sulfhydryls

Original Robert's cytoprotection/adaptive cytoprotection/ organoprotection concept elaborated with standard cytoprotective agents/mediators, prostaglandins, somatostatin, sulfhydryls

Direct instillation into the stomach of various highly damaging agents (i.e., absolute ethanol) or application of NSAIDs as the initial background

The Robert's cytoprotection/adaptive cytoprotection/organoprotection concept as the wound-healing concept

Concept of cytoprotection holds stomach cell protection in order to maintain stomach integrity against various noxious agents (Robert's killing cell by contact) - induced by direct instillation into the stomach of various highly damaging agents (i.e., absolute ethanol), or application of NSAIDs. ${ }^{14-19}$ The next extension of the concept means the generalization of the stomach cell protection to the innate cell protection in the other organs (stomach cytoprotection $\rightarrow$ organoprotection). ${ }^{20,21}$ Thus, in subsequent order, generalization from stomach epithelia to other epithelia appears, i.e., skin, liver, pancreas, heart, etc., and thereby, the wound-healing concept (providing that the stomach ulcer is much like the skin ulcer a wound that from the unknown reason fail to heal), leading to the axiomatic statement "the healing is always the same." ${ }^{30}$

Less emphasized, but essentially important is the final point in the original cytoprotection concept, the continuous presentation of the cytoprotective agent in gastric mucosa, and likely in the gastric juice, and thereby, permanently maintaining stomach mucosa integrity. ${ }^{14-19}$ This however, directly holds that cytoprotective agent would be effective prophylactically (given before injury able to protect stomach mucosa) as well as therapeutically (given after injury, with the pre-existing lesion, able to rescue stomach mucosa). ${ }^{11}$ This is however not the case with standard agents (i.e., prostaglandins, somatostatin, sulfhydryls); they are effective only when given before injury, and thereby, less likely to play the role of the endogenous cytoprotective agent in permanent maintenance of the stomach and gastrointestinal tract integrity.

\section{Original Robert's cytoprotection/adaptive cytoprotection/ organoprotection concept elaborated with BPC 157 as prototype of cytoprotective agents/mediators}

\section{Pleotropic beneficial activity}

Unlike the standard agents (i.e., prostaglandins, somatostatin, sulfhydryls) (in)activity, ${ }^{11}$ the stable gastric pentadecapeptide BPC 157 actually satisfies these full activity criteria completely. ${ }^{11}$ Essentially stable and native in human gastric juice, BPC 157 is highly effective prophylactically and therapeutically in counter- 
acting various lesions in the whole gastrointestinal tract. ${ }^{1-13}$ This effectiveness accordingly includes distinctive ways of administration, acutely and chronically (i.e., bolus application intraperitoneal, intravenous, intragastric, enema, local bath, and continuous application in drinking water; local gel or cream). ${ }^{1-13}$ An interesting point is the local cream application in wound studies (local cream application heals deep skin burns much like stress gastric lesions in mice). ${ }^{1-13}$ As a real mediator of the Robert's cytoprotection, in addition to the stomach and gastrointestinal lesions, it has a pleotropic beneficial activity, and counteracts also the lesions in the other organs, liver, pancreas, lung, hearth and brain. ${ }^{1-13}$ Of particular notation in BPC 157 studies is that each organ lesion counteraction includes different noxious procedures but regularly the same dose regimen which enables to combine the beneficial effects consistently obtained. ${ }^{1-13}$ Illustratively, beneficial activity in heart assay includes counteraction of the doxorubicin cardiomyopathy, ${ }^{41}$ distinctive arrhythmias induced by digitalis, ${ }^{42}$ hyperkalemia, ${ }^{43}$ hypokalemia, ${ }^{44}$ succinylcholine, ${ }^{45}$ bupivacaine, ${ }^{46}$ and neuroleptics. ${ }^{47}$

\section{Pleotropic beneficial activity based on the opposing rela- tionship with alcohol and NSAIDs adverse effects}

In general cytoprotective/adaptive cytoprotection/organoprotection terms, and pleotropic range of the beneficial activities of BPC 157, and its opposing relationship with the alcohol and NSAIDs adverse effects, already reviewed, ${ }^{8,11}$ this extension is quite logical. The major argument is that the Robert's standard noxious agents, alcohol and NSAIDs, used as the prototype noxious agents in the initial demonstration of the cytoprotection lesions in the stomach, have pleotropic damaging activity. ${ }^{8,11}$ Alcohol and NSAIDs would also regularly induce various lesions in the other organs as well. ${ }^{11}$ Vice versa, it is likely that if cytoprotective agents induce the innate counteraction of the initial Robert-lesions (alcohol, NSAIDs) in the stomach, the same agent exhibits an alike counteraction of the other lesions regularly induced by alcohol and NSAIDs (i.e., in liver, brain). ${ }^{11}$ As we reported, this special combined point that should characterize the effects of any suited cytoprotective agent in practice fulfils the corresponding range of beneficial effect of BPC 157. We noted the antagonization of the corresponding alcoholinduced $^{48-52}$ and NSAIDs-induced ${ }^{53-57}$ stomach lesions, much like the counteraction of the liver and brain lesions, which were induced by alcohol ${ }^{58-60}$ or NSAIDs. ${ }^{53-57,61}$ To emphasize, much like the prototypic acute alcohol-stomach lesion, ${ }^{48-51}$ the beneficial range of the BPC 157 counteracts alcohol in both acute intoxication (protracted drowsiness, increased alcohol level in blood, hypothermia) and chronic intoxication (withdrawal convulsions). ${ }^{59,60}$ Likewise, it counteracts consequences of the chronic abuse (drinking alcohol for 3 months) ${ }^{58}$ such as chronic gastric lesions ${ }^{51}$ or liver lesions with portal hypertension. ${ }^{58}$ For NSAIDsadverse effects (i.e., gastrointestinal, liver and brain injuries), BPC 157 counteraction includes both COX-1 and COX-2 block- ers. ${ }^{52-57,61}$ BPC 157 reduces bleeding after amputation, and may also counteract aspirin induced prolongation of bleeding and thrombocytopenia. ${ }^{62,63}$ An interesting point is that BPC 157 may also prevent and reverse adjuvant arthritis in rats. ${ }^{64}$ Thus, unlike mentioned standard agents, BPC 157 achieves stomach cytoprotection $\rightarrow$ organoprotection. ${ }^{11}$

An important point is that this innate stability (as mentioned, resistant to degradation in human gastric juice more than 24 hours) provides the advantage of the administration using various routes of applications. ${ }^{1-13}$

\section{BPC 157 with the stomach cytoprotection concept $\rightarrow$ wound healing concept}

In addition, the other point considers the stomach cytoprotection concept $\rightarrow$ wound healing concept. ${ }^{1}$ Note, the original cytoprotective agenda (with the generalized cell protection, within the same "cytoprotective" background) includes the improved healing in different tissues. ${ }^{20,21}$ Indicatively, BPC 157 improves the healing of the skin wounds, ${ }^{65-68}$ muscle, ${ }^{69-73}$ tendon, ${ }^{73-77}$ ligament, ${ }^{78}$ and bone injuries. ${ }^{79-81}$ Furthermore, the observed considerable recovery of the skin wound and muscle, tendon, ligament and bone, after severe injury that could be not spontaneously healed ${ }^{65-81}$ may be quite indicative. These healing processes may suggest that BPC 157 may distinctively affect tissue healing (i.e., with BPC 157, tendon heals with the tendon, not with the bone detached rat Achilles tendon from calcaneus would be properly reattached to calcaneus without surgical intervention). ${ }^{73-77}$ Thus, we suggested that BPC 157 exerts its effect simultaneously in the healing of different tissues, and accommodate the healing processes in different tissues. ${ }^{1}$ As a supportive analogy a clear demonstration of the rat fistulas healing appears, ${ }^{82-87}$ and thereby simultaneously achieves healing in different tissues. In rat fistulas studies, ${ }^{82-87}$ creation of the fistulas by anastomoses between the different tissues, accurately provides the defined defect in each of the tissues. Due to the comparative small size of the rats and large size of the defects, these defects would fairly mimic large fistulas in patients that would hardly heal spontaneously. ${ }^{82-87}$ A similar demonstration should be the creation of the different gastrointestinal anastomoses ${ }^{57,88-92}$ that would accordingly heal as opposed to commonly poor healing in the corresponding control rats. Thus, the healing of the esophagocutaneous, ${ }^{82}$ gastrocutaneous, ${ }^{83}$ duodenocutaneous, ${ }^{84}$ colocutaneous, ${ }^{85}$ vesicovaginalis, ${ }^{86}$ and rectovaginalis fistulas, ${ }^{87}$ assessed grossly, biomechanically and microscopically means demonstration of the both external and internal fistulas healing ${ }^{82-87}$ and consequently, the realization of the simultaneous healing of different tissues. Likewise, is the demonstration of the improved anastomoses healing, ${ }^{57,88-92}$ gastrointestinal (i.e., esophagogastric, esophagojejunal ileoileal, jejunoileal, colon anastomoses), ${ }^{57,88-92}$ but also nerve (sciatic nerve) ${ }^{93}$ and vascular (abdominal aorta). ${ }^{94}$ Consequently, the evidence that it may close and heal the various fistulas ${ }^{82-87}$ and improve anastomosis healing, ${ }^{57,88-94}$ may be 
a proof of the fulfilled wound healing concept.

\section{BPC 157 stomach cytoprotection concept $\rightarrow$ wound heal- ing concept with respect to angiogenesis and distinction from the standard angiogenic growth factors}

The other generalization of the stomach cytoprotection concept $\rightarrow$ wound healing concept is the application of standard peptidergic growth factors (providing that they are mediators of healing) in both gastrointestinal tract healing (i.e., ulceration healing) and skin wound and soft tissue injuries (i.e., muscle, tendon, ligament, bone healing). ${ }^{1,30}$ In particular, this is due to the effect on the angiogenesis as a common healing point. ${ }^{30} \mathrm{~A}$ careful comparison between BPC 157 and standard angiogenic growth factors provides the convincing evidence in favor of BPC 157 over standard angiogenic growth factors. ${ }^{1}$ First, BPC 157 significantly exceeds regular anti-ulcer agents and their effect on angiogenesis. ${ }^{95}$ Second, its angiogenic response ${ }^{1-13}$ is quite special, it integrates its healing assets ${ }^{73}$ and a number of molecular pathways. ${ }^{2,96-101}$ Illustratively, BPC 157 heals corneal ulcer and lesions and maintains corneal transparency. ${ }^{102,103}$ Third, BPC 157 exerts its effect within the same dose range in healing of the lesions in the whole gastrointestinal tract, as well as the healing of the skin wound, the healing of the severely injured (including transection) muscle, tendon, ligament, and finally bone healing (including pseudoarthrosis healing). ${ }^{1-13}$ Contrarily, the standard angiogenic growth factors have limited effect in gastrointestinal tract, and their effect could be different from one part to another. ${ }^{1}$ Especially, for the skin wound, muscle, tendon, ligament and bone healing they obviously need various carriers in addition, and thereby, they could exert mostly a local effect. ${ }^{1}$ Besides, standard angiogenic growth factors need a different carrier addition, and together may have uncertain activity attribution (peptide; carrier; peptide+carrier complex). ${ }^{1}$ Unlike this common negative point for the standard agents application, BPC 157 always given alone, acts alone, and therefore, all of the effects could be clearly described to its own peptide activity. ${ }^{1-13}$ In addition, the important point is that BPC 157 may induce also nerve healing, peripherally ${ }^{93}$ and centrally. $^{104}$

Such clear distinction in angiogenesis is essential (and thereby, "controlling of angiogenesis") ${ }^{1}$ and regular with BPC $157 .{ }^{1}$ In particular, this should be emphasized since alike distinction is unusual for the standard growth factors, ${ }^{1}$ and thereby (angiogenesis/healing/tumor promoting), the fear of tumor promoting known with standard growth factors. Contrarily, "controlling of angiogenesis" is along with an increased angiogenesis to promote healing, ${ }^{73}$ an increased expression of vascular endothelial growth factor (VEGF) and subsequent pathways, ${ }^{2,96-101}$ and as study of Kang et al., ${ }^{2}$ documented, BPC 157 fully counteracted tumor cachexia, and prolonged survival, counteracted increase of the pro-cachectic and pro-inflammatory cytokines, and related molecular pathways effects. ${ }^{2}$ In addition, a study showing that BPC 157 actually counteracted tumor promoting effect of VEGF should be also mentioned. ${ }^{105}$

This point was already elaborated especially in our shortbowel syndrome study. ${ }^{91}$ In rats which underwent large resection of the small bowel, BPC 157 leads to the balanced adaptation of all three intestinal wall layers, resulting in normal weigh achievement, and attenuation of the remote organs damages (i.e., liver and brain), unlike rapidly presented and progressed shortbowel syndrome. . $^{57,91}$

\section{The concept of cytoprotection as the injury of the stomach endothelium that would precede and induce stomach epithelia damage and BPC 157 "stomach endothelium protection" to the protection of the endothelium of other vessels}

The next essential point of the concept of cytoprotection is the injury of stomach endothelium that would precede and induce the stomach epithelia damage, and thereby stomach endothelium protection to protect stomach epithelia as an innate effect of the cytoprotective agents. ${ }^{106-109}$ While the originally noted endothelium protection remains quite strictly confined to the stomach endothelium protection, ${ }^{106-109}$ a point we described, is that this means generalization of the "stomach endothelium protection" to the protection of the endothelium of other vessels. ${ }^{1-13}$ Thus, with BPC 157 use in the resolving of the cytoprotection concept, its local in the stomach to its systemic concept terms, this combined effect could envisage its controlling of endothelium maintenance (as before the stomach cell protection) to be suited for an alike therapy generalization. ${ }^{1-13}$ This point was illustrated by the evidence obtained in rats in which abdominal aorta anastomosis (and thereby arterial thrombosis), ${ }^{94}$ or inferior caval vein occlusion (and thereby venous thrombosis), ${ }^{101}$ or amputation and anticoagulants application (and thereby prolonged bleeding and thrombocytopenias) ${ }^{63,64}$ were present. Specifically, in rats, with the anastomosed abdominal aorta, thrombosis was either prevented, or once established, reversed. ${ }^{94}$ In rats, with the ligation of the inferior caval vein, direct vein injury and thrombosis were counteracted (occlusion up to the right ovarian vein leading to vessel injury, stasis, thrombosis and hemodynamic changes a recapitulation of Virchow). ${ }^{101}$ In rats with tail or foot amputation, and heparin, warfarin and aspirin application, without ${ }^{62}$ or with nitric oxide (NO)-agents, NO synthase (NOS)-blocker N-nitro-L-arginine methyl ester (LNAME) or NOS-substrate L-arginine, ${ }^{63}$ prolonged bleeding and thrombocytopenia were counteracted.$^{62,63}$ Likewise, in rats with inferior caval vein-ligation thrombocytopenia and prolonged bleeding were all counteracted. ${ }^{101}$

Finally, a general argumentation within the commonly accepted concepts, holds that the wound healing process accomplished all of the four major events (vascular constriction, loose platelet plug, fibrin mesh to ensure stability of platelet plug, dissolution of the clot) that occur in a set order following the 
loss of vascular integrity. ${ }^{62}$ Thereby, an agent implemented in wound healing, ${ }^{65-81}$ such as stable gastric pentadecapeptide BPC 157 , has shown to be effective in wound healing but also effective in bleeding disorders. ${ }^{62}$

\section{Stable gastric pentadecapeptide BPC 157-NO-system- relation}

For effective application of the background of BPC 157, of particular significance should be BPC 157 interaction with NOsystem. This interaction seems to be always present, while the relevance could be high knowing general importance of NOsystem, and providing a clear demonstration of the interaction between BPC 157 and the whole NO-system in various models and species. ${ }^{1-13}$ The data so far presented suggested that BPC 157 may modulate NO-system effect, providing that its effectiveness could override the effects of NOS-blockade (induced by NOS-blocker L-NAME), NOS-overstimulation (induced by NOSsubstrate L-arginine application), or NOS-system immobilization (induced by simultaneous administration of L-NAME and Larginine). ${ }^{1-13}$ The point of homeostasis that may be induced by BPC 157 was shown using the example of L-NAME hypertension, and L-arginine-hypotension, both counteracted with BPC 157 application (while BPC 157 has no influence on normal blood pressure). ${ }^{49}$

More specifically, stable gastric pentadecapeptide BPC 157NO-system-relation could suggest its close participation in Moncada's (maintained vascular integrity, platelets control) homeostatic healing response of NO-system to injury. ${ }^{7}$ Seen from the viewpoint of the BPC 157-NO-system-relation, it is important that BPC 157's particular healing effect also affects all events after the loss of vascular integrity. ${ }^{7}$ Dependent on circumstances, it reduces either thrombosis (abdominal aorta anastomosis; inferior caval vein occlusion ${ }^{101}$ ) or bleeding/thrombocytopenia (amputation; ${ }^{62,63}$ heparin, warfarin, aspirin; $;^{62,63}$ after prolonged period of inferior caval vein occlusion ${ }^{101}$ ). Furthermore, in a series of different injurious models, acute and chronic, ${ }^{1-13}$ BPC 157 consistently promotes healing after severe injuries in various tissues which are spontaneously unable to heal $;^{1-13}$ stimulates egr-1 and naB2 genes and different pathways; ${ }^{96-101}$ exhibits high safety (LD1 not achieved). ${ }^{1-13}$ Thus, a hypothesis is that BPC 157 (since it is formed constitutively in the gastric mucosa, stable in human gastric juice, along with significance of NO-synthase and the basal formation of NO in stomach mucosa, greater than that seen in other tissues) exhibits a general, effective competition to both L-arginine analogues (i.e., L-NAME) and L-arginine. ${ }^{1-13}$ Furthermore, this has some physiologic importance (i.e., controlled NO-generation, providing that in the stomach BPC 157 induced NO-released opposed L-arginine-induced NO-release, and that BPC 157 induced NOreleased is completely resistant to L-NAME application unlike Larginine). ${ }^{49}$ It practically supports its beneficial effects illustrating BPC 157 and NO-system mutual (with L-NAME/L-arginine; alone and together) relations. The next concluding arguments are the subset of the evidence obtained in gastric mucosa and mucosal protection, following alcohol lesions, in cytoprotection course, NO-generation, and blood pressure regulation. ${ }^{49}$ Furthermore, the evidence includes NSAIDs gastrointestinal, liver, and brain lesions, failed anastomosis, and intestinal adaptation against deterioration after intestine resection. ${ }^{57,96-99}$ Alcohol acute/chronic intoxication, and withdrawal combined together, may be particular illustration ${ }^{59,60}$ much like the antagonization of thiopental anesthetic effect. ${ }^{110}$ Cardiovascular disturbances, chronic heart failure, pulmonary hypertension, and arrhythmias appear as a particular point of counteraction, much like the disturbances after hypokalemia and hyperkalemia, ${ }^{41-45}$ and potassium-cell membrane dysfunction or complex muscle disorders produced by potassium ${ }^{43-45}$ or magnesium overload, ${ }^{111}$ or pupil paralysis with atropine. ${ }^{112}$ Finally, the BPC 157-NOsystem-relation includes complex healing failure, proved by the fistulas healing, ${ }^{82,84-86}$ or particular anastomoses healing ${ }^{88}$ or vessel occlusion ${ }^{101,113}$ or cytostatic agents application (i.e., doxorubicin, cyclophosphamide). ${ }^{41,114}$ The notation that BPC 157-NOsystem-relation interferes with the effects of neuroleptics merits further elaboration. ${ }^{115,116}$ Finally, BPC 157 application lead to normalization of NO-level in tissues as well as counteraction of free-radicals formation. ${ }^{113,114,116}$ of note, how this advantage of modulating NO-system (i.e., particular effect on eNOS gene), may be a practical background which could be further translated into an enhanced clinical performance. ${ }^{1-13}$

\section{BPC 157 may have a particular additional effect during the injury on the blood vessel activity: vessel recruitment to circumvent the vessel occlusion}

The final argumentation goes to the findings that BPC 157 may have a particular additional effect during the injury on the blood vessel activity, much like vessel recruitment to circumvent the vessel occlusion, presenting an additional shunting and rapid bypassing loops to rapidly reestablish blood flow integrity. ${ }^{101,113,117,118}$ The particular illustration to damage counteraction appears with the ischemic/reperfusion colitis, ${ }^{113}$ and rapid bypassing loop through arcade vessels. ${ }^{113}$ Consequently, circumventing blockades, blood flow was restored, and pale areas without mucosal folds did not occur. ${ }^{113}$ Similar evidence follows the ligation of the superior anterior pancreaticoduodenal vein and duodenal congestion lesions, which were completely counteracted by BPC 157 application, both with intraabdomina bath or intragastric application with rapid presentation of the bypassing pathway through inferior anterior pancreaticoduodenal vein to superior mesenteric vein. ${ }^{117}$ This corresponds to the general evidence that BPC 157 was used in ulcerative colitis trial and duodenal lesions counteraction. ${ }^{1-13}$ As a proof of the integrative healing evidence (rapid cytoprotective endothelium rescue that BPC 157 exerted may be useful against damaging chain of events during ischemia [two ligations] and during 
reperfusion [ligations removed]) appear normalized NO- and malondialdehyde (MDA)-values in colon tissues, oxidative stress markers. ${ }^{113}$ On the other hand, with ligation of the inferior caval vein occlusion we made a recapitulation of Virchow. A consistent model goes with ligation leading to vessel injury, exposure of tissue factors, stasis, thrombosis, hemodynamic changes, arterial hypotension, and in particular, venous hypertension, which approximates values over $20 \mathrm{~mm} \mathrm{Hg}$ in the inferior caval vein, ${ }^{101}$ thereby being roughly four times higher than those in normal rats. All these adverse effects were counteracted by BPC 157 application. ${ }^{101}$ Interestingly, after cecum perforation, with BPC 157 administration we documented the gross reappearance of the vessels (USB micro camera) quickly propagating toward the defect at the caecum surface, defect in contraction, bleeding attenuation, MDA- and NO-levels normalized in colon tissue at 15 minutes, and advanced healing of colon lesions and less adhesions at days 1 and 7. ${ }^{118}$

\section{Robert's stomach cytoprotection concept as the peripheral part of the brain-gut axis as beneficial background for the BPC 157 application in various CNS-disorders}

Besides, as we reviewed, ${ }^{5}$ long ago, the Robert's stomach cytoprotection concept was considered as the peripheral part of the brain-gut axis. ${ }^{119}$ The most prominent action of many peptides (i.e., bombesin, thyrotropin-releasing hormone, CRF, neurotensin) on gastric lesions ${ }^{120-128}$ which appears when applied within the specific hypothalamic and brain stem sites or into the cerebrospinal fluid, ${ }^{128}$ requires a corresponding effect from periphery.

And thereby, the beneficial background for the BPC 157 application in various central nervous system (CNS)-disorders holds the commonly accepted knowledge that brain-gut interaction involves, among others, gut-brain axis and interaction, peptidergic growth factors which are native in gastrointestinal tract and have strong antiulcer potency and thus could from periphery beneficially affect CNS-disorders. ${ }^{5}$ Initially, corresponding beneficial central influence on serotonin system was seen when BPC 157 was given peripherally and a serotonin release in particular brain areas, mostly nigrostriatal, was changed and markedly increased, ${ }^{129}$ Porsolt's test and chronic stress induced immobility reduced more than after imipramine administration while serotonin syndrome (irreversible monoamine oxidase [MA0]-inhibition+serotonin precursor as serotonin substrate) was fully counteracted. ${ }^{130,131}$ Influence on dopamine system goes with the counteraction of the adverse effects of the amphetamine, 1-methyl-4-phenyl-1,2,3,6-tetrahydrophyridine (MPTP; Parkinson's model in mice), reserpine, haloperidol and other dopamine antagonists. ${ }^{38,115,116,131,132}$ Thus, BPC 157 does not act as a serotonin substrate. ${ }^{129}$ BPC 157 does inhibit MAO-inhibition, ${ }^{131}$ modulates serotonergic and dopaminergic systems, ${ }^{38,115,116,129-136}$ beneficially affects various behavioral disturbances that otherwise appeared due to specifically (over)stimulated/damaged neurotransmitters systems. ${ }^{5}$

\section{Dopamine system as an additional background for Robert's stomach cytoprotection. BPC 157 modulates dopamine system activity}

This assumption follows the evidence that the dopamine system provides an additional background also for Robert's stomach cytoprotection ${ }^{13,137-141}$ and gastric lesions appear after dopamine antagonists, MPTP, and reserpine. ${ }^{132,133,135}$ Thus, for BPC 157 application as a cytoprotective agent, we could likely envisage an analogy with the counteraction of the stomach lesion induced by NSAIDs much like the counteraction of all others adverse of NSAIDs effects, including the central disturbances. Accordingly, BPC 157 may, in addition to gastric lesions induced by dopamine antagonists, MPTP, and reserpine, counteract also their central disturbances such as akinesia, catalepsy as well as prolonged QTc intervals or sphincter malfunction in periphery. ${ }^{38,115,116,129-135}$ Thus, it may be that BPC 157 does modulate dopamine system activity. A supporting point for such particular modulatory effect is the evidence that BPC 157 counteracts also amphetamine both acute and chronic toxicity. ${ }^{38,115,116,129-135}$

\section{BPC 157 neuroprotective effects}

In addition, BPC 157 has neuroprotective effects. It protects somatosensory neurons in capsaicin-treated rats. ${ }^{53}$ Sciatic nerve regeneration appears after transection with BPC 157 application, local (into the tube), intragastric or intraperitoneal. ${ }^{93}$ After traumatic brain injury, it counteracts the otherwise progressing course. ${ }^{104}$ In rat spinal cord compression with tail paralysis, axonal and neuronal necrosis, demyelination, cyst formation, BPC 157 result in marked recovery and rescues tail function in both short-terms and long-terms. ${ }^{142}$ After NSAIDs ${ }^{54-57,61}$ or insulin overdose ${ }^{143}$ or cuprizone (a neurotoxin that induces multiple sclerosis like lesion in rat model ${ }^{92}$ or large bowel resection, ${ }^{57}$ encephalopathies were attenuated along with gastrointestinal, liver and vascular injuries. When convulsions appeared with paracetamol ${ }^{61}$ or insulin, ${ }^{141}$ they were completely eliminated or markedly attenuated, ${ }^{61,141}$ much like muscle weakness in cuprizone-rats. ${ }^{92}$

\section{BPC 157 interacts with several molecular pathways}

Finally, as a conclusive point, BPC 157 interacts with several molecular pathways as it was demonstrated by many other groups as well..$^{96-101}$ The wide range of the implicated pathways and thereby the functions known to be mediated by those pathways may be certainly a consistent background for the wide range of the BPC 157 claimed in our elaboration of the cytoprotection concept application in therapy. In this, of particular importance may be the resolving evidence that BPC 157 counteracted tumor cachexia. Along with the counteracted increase of pro-inflammatory cytokines such as interleukin-6, tumor necrosis factor- $\alpha$, looking at muscle metabolism relevant to cance 
cachexia, BPC 157 counteracted muscle wasting, significantly corrected deranged muscle proliferation as well as myogenesis, the changes in the expression of transcription factor (Fox03a), phospho-AKT (p-AKT), phospho mammalian target of rapamycin (p-mTOR), and phospho glycogen synthase kinase $3 \beta$ (p-GSK$3 \beta){ }^{2}$ Of note, BPC 157 recovery muscle and muscle function occurs upon direct traumas (that would result in the severe injuries that would be hardly healed spontaneously). ${ }^{69-73}$ Likewise, it occurs after vascular occlusion, ${ }^{94}$ neuromuscular blockade (succinylcholine application), ${ }^{45}$ potassium or magnesium overload, ${ }^{43,11}$ dopamine system failure (nigrostriatal damage [1-methyl4-phenyl-1,2,3,6-tetrahydropyridine, MPTP]), ${ }^{130}$ vesicle depletion (reserpine), ${ }^{132}$ dopamine receptors blockade (haloperidol and other dopamine antagonists),,$^{47,116,135}$ or dopamine receptors supersensitivity (amphetamine after haloperidol) ${ }^{133}$ or dopamine over-release (amphetamine). ${ }^{133,134}$ In addition, BPC 157 recovers also smooth muscle functions, that is, sphincters ${ }^{144-147}$ (lower esophageal, pyloric, ${ }^{144-146}$ urinary, ${ }^{147}$ pupil ${ }^{112}$ ), upon direct injury (stretching), ${ }^{144-147}$ indirect injury (fistula, ${ }^{82-84}$ anastomosis, ${ }^{87}$ ) or damaging agents (alcohol, ${ }^{52}$ potassium or magnesium overload, ${ }^{43,111}$ NSAIDs, ${ }^{115}$ neuroleptics, ${ }^{116}$ NO-agents, ${ }^{112,116}$ atropine ${ }^{112}$ ) application. Thus, we could suggest that the pathway indicated in the successful counteraction of the tumor-induced cachexia and muscle wasting, ${ }^{2}$ may be the common key how BPC 157 may function.

\section{CONCLUSIONS}

As a general common point, the Robert's stomach cytoprotection/adaptive cytoprotection/organoprotection ${ }^{14-19}$ much like the Selye's stress coping response, ${ }^{22-25}$ both receive an increasing popularity, but limited practical applicability when largely confronted with humbler but more precise ideas. Since the very beginning, Robert's stomach cytoprotection/adaptive cytoprotection/organoprotection has been in the focus on the demonstration of the particular beneficial effect of the cytoprotectants, using prostaglandins as the prototype, but also other standard cytoprotectants. On that account, innate beneficial effect, Robert's stomach cytoprotection/adaptive cytoprotection/ organoprotection ${ }^{14-19}$ tolerates the lack of the full understanding of the involved mechanisms, specific cellular changes seldom identified, the surface epithelial cells not protected, electrophysiologic parameters (potential difference) instead macroscopic/ microscopic assessments. ${ }^{148}$ Thus, if the strength of the particular beneficial effect is the most important proof of the concept with the prostaglandins and other standard cytoprotectants, we have to admit that Robert's stomach cytoprotection/adaptive cytoprotection/organoprotection tolerates no recovery of the preexisting stomach lesions, providing only prophylactic effect. ${ }^{14-19}$ Also, there are very limited extension of the standard cytoprotectants effect from the stomach lesions to other organs lesions (liver, pancreas), ${ }^{20,21}$ thus a quite limited cytoprotective and organoprotective therapeutic potential.

Finally, Robert's concept has a methodology issue. ${ }^{53}$ It applies necrotizing agent (i.e., absolute alcohol) intragastrically, delivery goes directly through the inserted orogastric tube into the stomach (procedure that Robert [incorrectly] referred as ora application). ${ }^{14-19}$ Far from reality of the regular per-oral application, it means completely "unprepared stomach" to an unusual event. Contrarily, we recently demonstrated that the new initial target is the tongue, resulting in spontaneous reversal of strong alcohol-stomach lesions. ${ }^{53}$ BPC 157 therapy functions also within the redirected complexity of Robert's stomach cytoprotection/ adaptive cytoprotection concept. ${ }^{53}$

Likewise, the follow-up of the Robert's stomach cytoprotection/adaptive cytoprotection/organoprotection ${ }^{14-19}$ much like the Selye's stress coping response, ${ }^{22-25}$ done by BPC $157,,^{1-13}$ reveals that the extensive demonstration of the beneficial effects may have similar problems as original concepts with the less focused involved mechanisms. However, the achieved demonstration of the beneficial effects, and thereby stomach cytoprotection $\rightarrow$ organoprotection more reliable realization, involves the whole gastrointestinal tract, both prophylactic and therapeutic effect, including the pre-existing lesions in the most complex disturbances, such as the internal and external fistulas, or anastomosis complicated with severe colitis. ${ }^{1-13}$ There is a particular effect on endothelium integrity as well. ${ }^{1-13}$ Likewise, originally, BPC 157 effect was ascribed to its interaction and modulation of NO-system, while the interaction with prostaglandins, dopamine, and serotonin systems was also documented. ${ }^{1-13}$ BPC 157 also acts as a free radical scavenger, and normalizes NO- and MDA-levels in tissues, in ischemia and reperfusion. ${ }^{101,113,114,116-118}$ There are subsequent particular studies of other groups, ${ }^{2,96-101}$ confirmation of the our original findings. ${ }^{65-78}$ Pleiotropic effect involving distinctive receptors, VEGF receptor 2 (VEGFR2), growth hormone receptors, distinctive pathways, VEGFR2-AKT-eNOS (endothelial NOS), ERK $1 \frac{12}{2}$, focal adhesion kinase-paxillin, Fox03a, p-AKT, p-mTOR and p-GSK-3 $\beta$, distinctive loops, stimulation egr-1 gene and its corepressor gene naB2, counteraction of the increased pro-inflammatory and pro-cachectic cytokines, ${ }^{2,96-101}$ would likely minimize the inherent lack of full understanding of the mechanisms which may be involved. But more important is the practical indicative evidence that even with the majority of studies on BPC 157 done on rodents given injection of the supplement, there are considerable number of the studies, particularly in gastrointestinal research, intragastric application or per-oral application in drinking water, correspondingly effective. ${ }^{12,41,43,48,51,54,55,58,59,61-63,82-85,89,91-93,98,114,116,117,143-147,149-155}$ In reality, in particular along with its safe profile, LD-1 not achieved, and no adverse effects in clinical trials, ${ }^{1-13}$ this evidence suggests easy practical clinical application (Fig. 1). While introducing a new concept BPC 157 first strictly follows, and then, it considerably extends, with recently demonstrated recruitment of blood vessels to bypass vascular occlusion, ${ }^{101,113,117,118}$ the guidelines 
of classic of stomach cells/endothelium cytoprotection ${ }^{14-21}$ and Selye's concept of stress response against stress injury to reestablish disturbed homeostasis. ${ }^{22-25,40}$

Thus, in practice, these findings may be relevant to resolving Robert's cytoprotection/adaptive cytoprotection/organoprotection, ${ }^{14-21}$ and consequently, Selye's stress coping response, ${ }^{22-25,40}$ and practical applicability.

\section{CONFLICTS OF INTEREST}

No potential conflict of interest relevant to this article was reported.

\section{ORCID}

Predrag Sikiric

https://orcid.org/0000-0002-7952-2252

\section{REFERENCES}

1. Seiwerth S, Rucman R, Turkovic B, et al. BPC 157 and standard angiogenic growth factors: gastrointestinal tract healing, lessons from tendon, ligament, muscle and bone healing. Curr Pharm Des 2018;24:1972-1989.

2. Kang EA, Han YM, An JM, et al. BPC157 as potential agent rescuing from cancer cachexia. Curr Pharm Des 2018;24:1947-1956.

3. Sikiric P, Rucman R, Turkovic B, et al. Novel cytoprotective mediator, stable gastric pentadecapeptide BPC 157: vascular recruitment and gastrointestinal tract healing. Curr Pharm Des 2018;24:1990-2001

4. Sikiric P, Seiwerth S, Rucman R, et al. Stress in gastrointestinal tract and stable gastric pentadecapeptide BPC 157. Finally, do we have a solution? Curr Pharm Des 2017;23:4012-4028.

5. Sikiric P, Seiwerth S, Rucman R, et al. Brain-gut axis and pentadecapeptide BPC 157: theoretical and practical implications. Curr Neuropharmacol 2016;14:857-865.

6. Seiwerth S, Brcic L, Vuletic LB, et al. BPC 157 and blood vessels. Curr Pharm Des 2014;20:1121-1125.

7. Sikiric P, Seiwerth S, Rucman R, et al. Stable gastric pentadecapeptide BPC 157-NO-system relation. Curr Pharm Des 2014;20:1126-1135.

8. Sikiric P, Seiwerth S, Rucman R, et al. Toxicity by NSAIDs: counteraction by stable gastric pentadecapeptide BPC 157. Curr Pharm Des 2013;19:76-83.

9. Sikiric P, Seiwerth S, Rucman R, et al. Focus on ulcerative colitis: stable gastric pentadecapeptide BPC 157. Curr Med Chem 2012;19:126-132.

10. Sikiric P, Seiwerth S, Rucman R, et al. Stable gastric pentadecapeptide BPC 157: novel therapy in gastrointestinal tract. Curr Pharm Des 2011;17:1612-1632.

11. Sikiric P, Seiwerth S, Brcic L, et al. Revised Robert's cytoprotection and adaptive cytoprotection and stable gastric pentadecapeptide BPC 157: possible significance and implications for novel mediator. Curr Pharm Des 2010;16:1224-1234.

12. Sikiric P, Seiwerth S, Brcic L, et al. Stable gastric pentadecapeptide BPC 157 in trials for inflammatory bowel disease (PL-10, PLD-116, PL 14736, Pliva, Croatia): full and distended stomach, and vascular response. Inflammopharmacology 2006;14:214221.

13. Sikirić P, Petek M, Rucman R, et al. A new gastric juice peptide, BPC. An overview of the stomach-stress-organoprotection hypothesis and beneficial effects of BPC. J Physiol Paris 1993;87:313-327.

14. Robert A. Cytoprotection by prostaglandins. Gastroenterology 1979;77(4 Pt 1):761-767.

15. Robert A. Cytoprotection and prostaglandins. Klin Wochenschr 1986;64 Suppl 7:40-43.

16. Robert A, Nezamis JE. Histopathology of steroid-induced ulcers: an experimental study in the rat. Arch Pathol 1964;77:407-423.

17. Robert A, Nezamis JE, Lancaster C, Davis JP, Field SO, Hanchar AJ. Mild irritants prevent gastric necrosis through "adaptive cytoprotection" mediated by prostaglandins. Am J Physiol 1983;245:G113-G121.

18. Robert A, Nezamis JE, Lancaster C, Hanchar AJ. Cytoprotection by prostaglandins in rats: prevention of gastric necrosis produced by alcohol, $\mathrm{HCl}, \mathrm{NaOH}$, hypertonic $\mathrm{NaCl}$, and thermal injury. Gastroenterology 1979;77:433-443.

19. Lancaster C, Robert A. Intestinal lesions produced by prednisolone: prevention (cytoprotection) by 16,16-dimethyl prostaglandin E2. Am J Physiol 1978;235:E703-E708.

20. Szabo S, Usadel KH. Cytoprotection: organoprotection by somatostatin. Gastric and hepatic lesions. Experientia 1982;38:254256.

21. Robert A, Lum JT, Lancaster C, Olafsson AS, Kolbasa KP, Nezamis JE. Prevention by prostaglandins of caerulein-induced pancreatitis in rats. Lab Invest 1989;60:677-691.

22. Selye H. A syndrome produced by diverse nocuous agents. Nature 1936;138:32.

23. Selye H. Forty years of stress research: principal remaining problems and misconceptions. Can Med Assoc J 1976;115:53-56.

24. Selye H. The stress of life. New York: McGraw-Hill, 1956.

25. Selye H. First annual report on stress. Montreal: Acta Inc., 1951.

26. Hartman FA, Brownell KA. The hormone of the adrenal cortex. Science 1930;72:76.

27. Swingle WW, Pfiffner JJ. The revival of comatose adrenalectomized cats with an extract of the suprarenal cortex. Science 1930;72:75-76.

28. Mason HL, Myers CS, Kendall EC. The chemistry of crystalline substances isolated from the suprarenal gland. J Biol Chem 1936;114:613-631.

29. de Herder WW. Heroes in endocrinology: Nobel Prizes. Endocr Connect 2014;3:R94-R104.

30. Tarnawski AS, Ahluwalia A. Molecular mechanisms of epithelial regeneration and neovascularization during healing of gastric and esophageal ulcers. Curr Med Chem 2012;19:16-27. 
31. Selye H. Relation of the adrenal cortex to arthritis. Lancet 1946;250:942.

32. Hench PS, Kendall EC, Slocumb CH, Polley HF. The effect of a hormone of the adrenal cortex (17-hydroxy-11-dehydrocorticosterone; compound E) and of pituitary adrenocorticotropic hormone on rheumatoid arthritis. Proc Staff Meet Mayo Clin 1949;24:181-197.

33. Taché Y, Million M. Role of corticotropin-releasing factor signaling in stress-related alterations of colonic motility and hyperalgesia. J Neurogastroenterol Motil 2015;21:8-24.

34. Taché Y, Brunnhuber S. From Hans Selye's discovery of biological stress to the identification of corticotropin-releasing factor signaling pathways: implication in stress-related functional bowel diseases. Ann N Y Acad Sci 2008;1148:29-41.

35. Stengel A, Taché Y. CRF and urocortin peptides as modulators of energy balance and feeding behavior during stress. Front Neurosci 2014;8:52

36. Stengel A, Taché Y. Corticotropin-releasing factor signaling and visceral response to stress. Exp Biol Med (Maywood) 2010;235: 1168-1178.

37. Nakane T, Kanie N, Audhya T, Hollander CS. The effects of centrally administered neuropeptides on the development of gastric lesions in the rat. Life Sci 1985;36:1197-1203.

38. Filaretova LP, Bagaeva TR, Morozova OIu. Gastroprotective action of corticotropin-releasing factor (CRF): involvement of glucocorticoids and CRF receptors type 2. Ross Fiziol Zh Im I M Sechenova 2012;98:1555-1566.

39. Jones MT. The pituitary-adrenal axis. In: Parnham MJ, Bruinvels J, eds. Discoveries in pharmacology. Volume 2. Amsterdam: Elsevier, 1984:285-305.

40. Masson G, Selye H. Réaction générale d'adaptation: ses indications pratiques. Can J Comp Med 1938;2:282-285.

41. Lovric-Bencic M, Sikiric P, Hanzevacki JS, et al. Doxorubicinecongestive heart failure-increased big endothelin-1 plasma concentration: reversal by amlodipine, losartan, and gastric pentadecapeptide BPC157 in rat and mouse. J Pharmacol Sci 2004;95: 19-26.

42. Balenovic D, Bencic ML, Udovicic M, et al. Inhibition of methyldigoxin-induced arrhythmias by pentadecapeptide BPC 157: a relation with NO-system. Regul Pept 2009;156:83-89.

43. Barisic I, Balenovic D, Klicek R, et al. Mortal hyperkalemia disturbances in rats are NO-system related: the life saving effect of pentadecapeptide BPC 157. Regul Pept 2013;181:50-66.

44. Balenovic I, Barisic I, Prkacin I, et al. Mortal furosemide-hypokalemia-disturbances in rats NO-system related: shortened survival by L-NAME: therapy benefit with BPC 157 peptide more than with L-arginine. J Clin Exp Cardiolog 2012;3:201.

45. Stambolija V, Stambolija TP, Holjevac JK, et al. BPC 157: the counteraction of succinylcholine, hyperkalemia, and arrhythmias. Eur J Pharmacol 2016;781:83-91.

46. Zivanovic-Posilovic G, Balenovic D, Barisic I, et al. Stable gastric pentadecapeptide BPC 157 and bupivacaine. Eur J Pharmacol
2016;793:56-65

47. Strinic D, Belosic Halle Z, et al. BPC 157 counteracts QTc prolongation induced by haloperidol, fluphenazine, clozapine, olanzapine, quetiapine, sulpiride, and metoclopramide in rats. Life Sci 2017;186:66-79.

48. Sikiric P, Seiwerth S, Grabarevic Z, et al. The beneficial effect of BPC 157, a 15 amino acid peptide BPC fragment, on gastric and duodenal lesions induced by restraint stress, cysteamine and 96\% ethanol in rats: a comparative study with $\mathrm{H} 2$ receptor antagonists, dopamine promotors and gut peptides. Life Sci 1994;54:PL63PL68.

49. Sikirić P, Seiwerth S, Grabarević Z, et al. The influence of a novel pentadecapeptide, BPC 157, on N(G)-nitro-L-arginine methylester and L-arginine effects on stomach mucosa integrity and blood pressure. Eur J Pharmacol 1997;332:23-33.

50. Sikirić P, Seiwerth S, Desković S, et al. New model of cytoprotection/adaptive cytoprotection in rats: endogenous small irritants, antiulcer agents and indomethacin. Eur J Pharmacol 1999;364:23-31.

51. Prkacin I, Aralica G, Perovic D, et al. Chronic cytoprotection: pentadecapeptide BPC 157, ranitidine and propranolol prevent, attenuate and reverse the gastric lesions appearance in chronic alcohol drinking rats. J Physiol Paris 2001;95:295-301.

52. Becejac T, Cesarec V, Drmic D, et al. An endogeous defensive concept, renewed cytoprotection/adaptive cytoprotection: intra(per)-oral/intraastric strong alcohol in rat. Involvement of pentadecapeptide BPC 157 and nitric oxide system. J Physiol Pharmacol 2018;69:429-440.

53. Sikirić P, Seiwerth S, Grabarević Z, et al. Beneficial effect of a novel pentadecapeptide BPC 157 on gastric lesions induced by restraint stress, ethanol, indomethacin, and capsaicin neurotoxicity. Dig Dis Sci 1996;41:1604-1614.

54. Ilic S, Drmic D, Franjic S, et al. Pentadecapeptide BPC 157 and its effects on a NSAID toxicity model: diclofenac-induced gastrointestinal, liver, and encephalopathy lesions. Life Sci 2011;88:535542.

55. Ilic S, Drmic D, Zarkovic K, et al. Ibuprofen hepatic encephalopathy, hepatomegaly, gastric lesion and gastric pentadecapeptide BPC 157 in rats. Eur J Pharmacol 2011;667:322-329.

56. Drmic D, Kolenc D, Ilic S, et al. Celecoxib-induced gastrointestinal, liver and brain lesions in rats, counteraction by BPC 157 or L-arginine, aggravation by L-NAME. World J Gastroenterol 2017;23:5304-5312.

57. Lojo N, Rasic Z, Zenko Sever A, et al. Effects of diclofenac, LNAME, L-arginine, and pentadecapeptide BPC 157 on gastrointestinal, liver, and brain lesions, failed anastomosis, and intestinal adaptation deterioration in 24 hour-short-bowel rats. PLoS One 2016;11:e0162590.

58. Prkacin I, Separovic J, Aralicia G, et al. Portal hypertension and liver lesions in chronically alcohol drinking rats prevented and reversed by stable gastric pentadecapeptide BPC 157 (PL-10, PLD-116), and propranolol, but not ranitidine. J Physiol Paris 
2001;95:315-324.

59. Blagaic AB, Blagaic V, Romic Z, Sikiric P. The influence of gastric pentadecapeptide BPC 157 on acute and chronic ethanol administration in mice. Eur J Pharmacol 2004;499:285-290.

60. Boban-Blagaic A, Blagaic V, Romic Z, et al. The influence of gastric pentadecapeptide BPC 157 on acute and chronic ethanol administration in mice. The effect of $\mathrm{N}(\mathrm{G})$-nitro-L-arginine methyl ester and L-arginine. Med Sci Monit 2006;12:BR36-BR45.

61. Ilic S, Drmic D, Zarkovic K, et al. High hepatotoxic dose of paracetamol produces generalized convulsions and brain damage in rats: a counteraction with the stable gastric pentadecapeptide BPC 157 (PL 14736). J Physiol Pharmacol 2010;61:241-250.

62. Stupnisek M, Franjic S, Drmic D, et al. Pentadecapeptide BPC 157 reduces bleeding time and thrombocytopenia after amputation in rats treated with heparin, warfarin or aspirin. Thromb Res 2012;129:652-659.

63. Stupnisek M, Kokot A, Drmic D, et al. Pentadecapeptide BPC 157 reduces bleeding and thrombocytopenia after amputation in rats treated with heparin, warfarin, L-NAME and L-arginine. PLoS One 2015;10:e0123454

64. Sikiric P, Seiwerth S, Grabarevic Z, et al. Pentadecapeptide BPC 157 positively affects both non-steroidal anti-inflammatory agent-induced gastrointestinal lesions and adjuvant arthritis in rats. J Physiol Paris 1997;91:113-122.

65. Seiwerth S, Sikiric P, Grabarevic Z, et al. BPC 157's effect on healing. J Physiol Paris 1997;91:173-178.

66. Mikus D, Sikiric P, Seiwerth S, et al. Pentadecapeptide BPC 157 cream improves burn-wound healing and attenuates burn-gastric lesions in mice. Burns 2001;27:817-827.

67. Sikiric P, Seiwerth S, Mise S, et al. Corticosteroid-impairment of healing and gastric pentadecapeptide BPC-157 creams in burned mice. Burns 2003;29:323-334.

68. Bilic M, Bumber Z, Blagaic AB, Batelja L, Seiwerth S, Sikiric P. The stable gastric pentadecapeptide BPC 157, given locally, improves CO2 laser healing in mice. Burns 2005;31:310-315.

69. Staresinic M, Petrovic I, Novinscak T, et al. Effective therapy of transected quadriceps muscle in rat: gastric pentadecapeptide BPC 157. J Orthop Res 2006;24:1109-1117.

70. Pevec D, Novinscak T, Brcic L, et al. Impact of pentadecapeptide BPC 157 on muscle healing impaired by systemic corticosteroid application. Med Sci Monit 2010;16:BR81-BR88.

71. Novinscak T, Brcic L, Staresinic M, et al. Gastric pentadecapeptide BPC 157 as an effective therapy for muscle crush injury in the rat. Surg Today 2008;38:716-725.

72. Mihovil I, Radic B, Brcic L, et al. Beneficial effect of pentadecapeptide BPC 157 on denervated muscle in rats. Proceedings of the International Congress of Myology; 2008 May 26-30; Marseille, France.

73. Brcic L, Brcic I, Staresinic M, Novinscak T, Sikiric P, Seiwerth S. Modulatory effect of gastric pentadecapeptide BPC 157 on angiogenesis in muscle and tendon healing. J Physiol Pharmacol 2009;60 Suppl 7:191-196.
74. Staresinic M, Sebecic B, Patrlj L, et al. Gastric pentadecapeptide BPC 157 accelerates healing of transected rat Achilles tendon and in vitro stimulates tendocytes growth. J Orthop Res 2003;21:976983.

75. Krivic A, Majerovic M, Jelic I, Seiwerth S, Sikiric P. Modulation of early functional recovery of Achilles tendon to bone unit after transection by BPC 157 and methylprednisolone. Inflamm Res 2008;57:205-210

76. Krivic A, Anic T, Seiwerth S, Huljev D, Sikiric P. Achilles detachment in rat and stable gastric pentadecapeptide BPC 157: promoted tendon-to-bone healing and opposed corticosteroid aggravation. J Orthop Res 2006;24:982-989.

77. Krivic A, Sikiric P. Comment on "Use of recombinant human bone morphogenetic protein-2 to enhance tendon healing in a bone tunnel”. Am J Sports Med 2003;31:636-637.

78. Cerovecki T, Bojanic I, Brcic L, et al. Pentadecapeptide BPC 157 (PL 14736) improves ligament healing in the rat. J Orthop Res 2010;28:1155-1161.

79. Sebecić B, Nikolić V, Sikirić P, et al. Osteogenic effect of a gastric pentadecapeptide, BPC-157, on the healing of segmental bone defect in rabbits: a comparison with bone marrow and autologous cortical bone implantation. Bone 1999;24:195-202.

80. Keremi B, Lohinai Z, Komora P, et al. Antiinflammatory effect of BPC 157 on experimental periodontitis in rats. J Physiol Pharmacol 2009;60 Suppl 7:115-122.

81. Gamulin 0, Serec K, Bilić V, et al. Monitoring the healing process of rat bones using Raman spectroscopy. J Mol Struct 2013;1044:308-313.

82. Cesarec V, Becejac T, Misic M, et al. Pentadecapeptide BPC 157 and the esophagocutaneous fistula healing therapy. Eur J Pharmacol 2013;701:203-212.

83. Skorjanec S, Dolovski Z, Kocman I, et al. Therapy for unhealed gastrocutaneous fistulas in rats as a model for analogous healing of persistent skin wounds and persistent gastric ulcers: stable gastric pentadecapeptide BPC 157, atropine, ranitidine, and omeprazole. Dig Dis Sci 2009;54:46-56.

84. Skorjanec S, Kokot A, Drmic D, et al. Duodenocutaneous fistula in rats as a model for "wound healing-therapy" in ulcer healing: the effect of pentadecapeptide BPC 157, L-nitro-arginine methyl ester and L-arginine. J Physiol Pharmacol 2015;66:581-590.

85. Klicek R, Sever M, Radic B, et al. Pentadecapeptide BPC 157, in clinical trials as a therapy for inflammatory bowel disease (PL14736), is effective in the healing of colocutaneous fistulas in rats: role of the nitric oxide-system. J Pharmacol Sci 2008;108:717.

86. Grgic T, Grgic D, Drmic D, et al. Stable gastric pentadecapeptide BPC 157 heals rat colovesical fistula. Eur J Pharmacol 2016;780:1-7

87. Baric M, Sever AZ, Vuletic LB, et al. Stable gastric pentadecapeptide BPC 157 heals rectovaginal fistula in rats. Life Sci 2016;148: 63-70.

88. Djakovic Z, Djakovic I, Cesarec V, et al. Esophagogastric anasto- 
mosis in rats: Improved healing by BPC 157 and L-arginine, aggravated by L-NAME. World J Gastroenterol 2016;22:9127-9140.

89. Sikiric P, Jadrijevic S, Seiwerth S, Sosa T, et al. Long-lasting cytoprotection after pentadecapeptide BPC 157, ranitidine, sucralfate or cholestyramine application in reflux oesophagitis in rats. $\mathrm{J}$ Physiol Paris 1999;93:467-477.

90. Vuksic T, Zoricic I, Brcic L, et al. Stable gastric pentadecapeptide BPC 157 in trials for inflammatory bowel disease (PL-10, PLD116, PL14736, Pliva, Croatia) heals ileoileal anastomosis in the rat. Surg Today 2007;37:768-777.

91. Sever M, Klicek R, Radic B, et al. Gastric pentadecapeptide BPC 157 and short bowel syndrome in rats. Dig Dis Sci 2009;54:20702083.

92. Klicek R, Kolenc D, Suran J, et al. Stable gastric pentadecapeptide BPC 157 heals cysteamine-colitis and colon-colon-anastomosis and counteracts cuprizone brain injuries and motor disability. J Physiol Pharmacol 2013;64:597-612.

93. Gjurasin M, Miklic P, Zupancic B, et al. Peptide therapy with pentadecapeptide BPC 157 in traumatic nerve injury. Regul Pept 2010;160:33-41.

94. Hrelec M, Klicek R, Brcic L, et al. Abdominal aorta anastomosis in rats and stable gastric pentadecapeptide BPC 157, prophylaxis and therapy. J Physiol Pharmacol 2009;60 Suppl 7:161-165.

95. Sikiric P, Separovic J, Anic T, et al. The effect of pentadecapeptide BPC 157, H2-blockers, omeprazole and sucralfate on new vessels and new granulation tissue formation. J Physiol Paris 1999;93:479-485.

96. Hsieh MJ, Liu HT, Wang CN, et al. Therapeutic potential of proangiogenic BPC157 is associated with VEGFR2 activation and up-regulation. J Mol Med (Berl) 2017;95:323-333.

97. Huang T, Zhang K, Sun L, et al. Body protective compound-157 enhances alkali-burn wound healing in vivo and promotes proliferation, migration, and angiogenesis in vitro. Drug Des Devel Ther 2015;9:2485-2499.

98. Chang CH, Tsai WC, Hsu YH, Pang JH. Pentadecapeptide BPC 157 enhances the growth hormone receptor expression in tendon fibroblasts. Molecules 2014;19:19066-19077.

99. Chang CH, Tsai WC, Lin MS, Hsu YH, Pang JH. The promoting effect of pentadecapeptide BPC 157 on tendon healing involves tendon outgrowth, cell survival, and cell migration. J Appl Physiol (1985) 2011;110:774-780.

100. Tkalcević VI, Cuzić S, Brajsa K, et al. Enhancement by PL 14736 of granulation and collagen organization in healing wounds and the potential role of egr-1 expression. Eur J Pharmacol 2007;570:212-221.

101. Vukojević J, Siroglavić M, Kašnik K, et al. Rat inferior caval vein (ICV) ligature and particular new insights with the stable gastric pentadecapeptide BPC 157. Vascul Pharmacol 2018;106:54-66.

102. Masnec S, Kokot A, Zlatar M, et al. Perforating corneal injury in rat and pentadecapeptide BPC 157. Exp Eye Res 2015;136:9-15.

103. Lazić R, Gabrić N, Dekaris I, Bosnar D, Boban-Blagaić A, Sikirić P. Gastric pentadecapeptide BPC 157 promotes corneal epithelial defects healing in rats. Coll Antropol 2005;29:321-325.

104. Tudor M, Jandric I, Marovic A, et al. Traumatic brain injury in mice and pentadecapeptide BPC 157 effect. Regul Pept 2010;160:26-32.

105. Radeljak S, Seiwerth S, Sikiric P. BPC 157 inhibits cell growth and VEGF signalling via the MAPK kinase pathway in the human melanoma cell line. Melanoma Res 2004;14:A14-A15.

106. Szabo S. Mechanism of mucosal protection. In: Hollander D, Tarnawski A, eds. Gastric cytoprotection: a clinician's guide. New York: Plenum Medical Book Co., 1989:49-90.

107. Szabo S, Trier JS. Pathogenesis of acute gastric mucosal injury: sulfhydryls as a protector, adrenal cortex as a modulator, and vascular endothelium as a target. In: Allen A, Flemstrom G, Garner A, Silen W, Turnberg LA, eds. Mechanism of mucosal protection in the upper gastrointestinal tract. New York: Raven, 1984:387-393.

108. Trier JS, Szabo S, Allan CH. Ethanol-induced damage to mucosal capillaries of rat stomach. Ultrastructural features and effects of prostaglandin F2 beta and cysteamine. Gastroenterology 1987;92:13-22.

109. Szabo S, Trier JS, Brown A, Schnoor J. Early vascular injury and increased vascular permeability in gastric mucosal injury caused by ethanol in the rat. Gastroenterology 1985;88(1 Pt 2):228-236.

110. Zemba M, Cilic AZ, Balenovic I, et al. BPC 157 antagonized the general anaesthetic potency of thiopental and reduced prolongation of anaesthesia induced by L-NAME/thiopental combination. Inflammopharmacology 2015;23:329-336.

111. Medvidovic-Grubisic M, Stambolija V, Kolenc D, et al. Hypermagnesemia disturbances in rats, NO-related: pentadecapeptide BPC 157 abrogates, L-NAME and L-arginine worsen. Inflammopharmacology 2017;25:439-449.

112. Kokot A, Zlatar M, Stupnisek M, et al. NO system dependence of atropine-induced mydriasis and L-NAME- and L-arginineinduced miosis: reversal by the pentadecapeptide BPC 157 in rats and guinea pigs. Eur J Pharmacol 2016;771:211-219.

113. Duzel A, Vlainic J, Antunovic M, et al. Stable gastric pentadecapeptide BPC 157 in the treatment of colitis and ischemia and reperfusion in rats: new insights. World J Gastroenterol 2017;23:8465-8488.

114. Luetic K, Sucic M, Vlainic J, et al. Cyclophosphamide induced stomach and duodenal lesions as a NO-system disturbance in rats: L-NAME, L-arginine, stable gastric pentadecapeptide BPC 157. Inflammopharmacology 2017;25:255-264.

115. Vitaic S, Stupnisek M, Drmic D, et al. Nonsteroidal anti-inflammatory drugs-induced failure of lower esophageal and pyloric sphincter and counteraction of sphincters failure with stable gatric pentadecapeptide BPC 157 in rats. J Physiol Pharmacol 2017;68:265-272.

116. Belosic Halle Z, Vlainic J, Drmic D, et al. Class side effects: decreased pressure in the lower oesophageal and the pyloric sphincters after the administration of dopamine antagonists, neuroleptics, anti-emetics, L-NAME, pentadecapeptide BPC 157 and 
L-arginine. Inflammopharmacology 2017;25:511-522.

117. Amic F, Drmic D, Bilic Z, et al. Bypassing major venous occlusion and duodenal lesions in rats, and therapy with the stable gastric pentadecapeptide BPC 157, L-NAME and L-arginine. World J Gastroenterol 2018;24:5366-5378.

118. Drmic D, Samara M, Vidovic T, et al. Counteraction of perforated cecum lesions in rats: effects of pentadecapeptide BPC 157, LNAME and L-arginine. World J Gastroenterol 2018;24:54625476.

119. Robert A, Saperas E, Zhang WR, et al. Gastric cytoprotection by intracisternal interleukin-1 beta in the rat. Biochem Biophys Res Commun 1991;174:1117-1124.

120. Taché Y, Kolve E, Maeda-Hagiwara M, Kauffman GL Jr. Central nervous system action of calcitonin to alter experimental gastric ulcers in rats. Gastroenterology 1988;94:145-150.

121. Taché Y. CNS peptides and regulation of gastric acid secretion. Annu Rev Physiol 1988;50:19-39.

122. Taché Y, Ishikawa T, Gunion M, Raybould HE. Central nervous system action of bombesin to influence gastric secretion and ulceration. Ann N Y Acad Sci 1988;547:183-193.

123. Taché Y, Maeda-Hagiwara M, Goto Y, Garrick T. Central nervous system action of TRH to stimulate gastric function and ulceration. Peptides 1988;9 Suppl 1:9-13.

124. Pappas TN, Welton M, Debas HT, Rivier J, Taché Y. Corticotropinreleasing factor inhibits gastric emptying in dogs: studies on its mechanism of action. Peptides 1987;8:1011-1014.

125. Gunion MW, Taché Y. Bombesin microinfusion into the paraventricular nucleus suppresses gastric acid secretion in the rat. Brain Res 1987;422:118-128.

126. Hernandez DE. Neuroendocrine mechanisms of stress ulceration: focus on thyrotropin-releasing hormone (TRH). Life Sci 1986;39:279-296.

127. Taché Y, Kolve E, Kauffman G. Potent CNS action of calcitonin to inhibit cysteamine-induced duodenal ulcers in rat. Life Sci 1987;41:651-655

128. Grijalva CV, Novin D. The role of the hypothalamus and dorsal vagal complex in gastrointestinal function and pathophysiology. Ann N Y Acad Sci 1990;597:207-222.

129. Tohyama Y, Sikirić P, Diksic M. Effects of pentadecapeptide BPC157 on regional serotonin synthesis in the rat brain: alphamethyl-L-tryptophan autoradiographic measurements. Life Sci 2004;76:345-357.

130. Sikiric P, Separovic J, Buljat G, et al. The antidepressant effect of an antiulcer pentadecapeptide BPC 157 in Porsolt's test and chronic unpredictable stress in rats. A comparison with antidepressants. J Physiol Paris 2000;94:99-104.

131. Boban Blagaic A, Blagaic V, Mirt M, et al. Gastric pentadecapeptide BPC 157 effective against serotonin syndrome in rats. Eur J Pharmacol 2005;512:173-179.

132. Sikiric P, Marovic A, Matoz W, et al. A behavioural study of the effect of pentadecapeptide BPC 157 in Parkinson's disease models in mice and gastric lesions induced by 1-methyl-4-phenyl- 1,2,3,6-tetrahydrophyridine. J Physiol Paris 1999;93:505-512.

133. Jelovac N, Sikirić P, Rucman R, et al. A novel pentadecapeptide, BPC 157, blocks the stereotypy produced acutely by amphetamine and the development of haloperidol-induced supersensitivity to amphetamine. Biol Psychiatry 1998;43:511-519.

134. Sikiric P, Jelovac N, Jelovac-Gjeldum A, et al. Pentadecapeptide BPC 157 attenuates chronic amphetamine-induced behavior disturbances. Acta Pharmacol Sin 2002;23:412-422.

135. Jelovac N, Sikiric P, Rucman R, et al. Pentadecapeptide BPC 157 attenuates disturbances induced by neuroleptics: the effect on catalepsy and gastric ulcers in mice and rats. Eur J Pharmacol 1999;379:19-31.

136. Boban Blagaic A, Turcic P, Blagaic V, et al. Gastric pentadecapeptide BPC 157 counteracts morphine-induced analgesia in mice. J Physiol Pharmacol 2009;60 Suppl 7:177-181.

137. Hernandez DE. Involvement of dopamine receptors in experimental ulceration. Int J Tissue React 1987;9:407-411.

138. Hernandez DE, Stanley DA, Melvin JA, Prange AJ Jr. Role of brain neurotransmitters on neurotensin-induced gastric cytoprotection. Pharmacol Biochem Behav 1985;22:509-513.

139. Orlando RC, Hernandez DE, Prange AJ Jr, Nemeroff CB. Role of the autonomic nervous system in the cytoprotective effect of neurotensin against gastric stress ulcers in rats. Psychoneuroendocrinology 1985;10:149-157.

140. Hernandez DE, Adcock JW, Orlando RC, Patrick KS, Nemeroff $\mathrm{CB}$, Prange AJ Jr. Prevention of stress-induced gastric ulcers by dopamine agonists in the rat. Life Sci 1984;35:2453-2458.

141. Sikiric P, Separovic J, Buljat G, et al. Gastric mucosal lesions induced by complete dopamine system failure in rats. The effects of dopamine agents, ranitidine, atropine, omeprazole and pentadecapeptide BPC 157. J Physiol Paris 2000;94:105-110.

142. Perovic D, Buljat G, Kolenc D, Drmic D, Seiwerth S, Sikiric P. Spinal cord injury in rat: therapeutic effect of pentadecapeptide BPC 157. FASEB J 2015;29(1 suppl):617.5.

143. Ilic S, Brcic I, Mester M, et al. Over-dose insulin and stable gastric pentadecapeptide BPC 157. Attenuated gastric ulcers, seizures, brain lesions, hepatomegaly, fatty liver, breakdown of liver glycogen, profound hypoglycemia and calcification in rats. J Physiol Pharmacol 2009;60 Suppl 7:107-114.

144. Petrovic I, Dobric I, Drvis P, et al. An experimental model of prolonged esophagitis with sphincter failure in the rat and the therapeutic potential of gastric pentadecapeptide BPC 157. J Pharmacol Sci 2006;102:269-277.

145. Dobric I, Drvis P, Petrovic I, et al. Prolonged esophagitis after primary dysfunction of the pyloric sphincter in the rat and therapeutic potential of the gastric pentadecapeptide BPC 157. J Pharmacol Sci 2007;104:7-18.

146. Petrovic I, Dobric I, Drmic D, et al. BPC 157 therapy to detriment sphincters failure-esophagitis-pancreatitis in rat and acute pancreatitis patients low sphincters pressure. J Physiol Pharmacol 2011;62:527-534.

147. Jandric I, Vrcic H, Jandric Balen M, et al. Salutary effect of gas- 
tric pentadecapeptide BPC 157 in two different stress urinary incontinence models in female rats. Med Sci Monit Basic Res 2013;19:93-102.

148. Szabó S. Critical and timely review of the concept of gastric cytoprotection. Acta Physiol Hung 1989;73:115-127.

149. Tlak Gajger I, Ribarić J, Smodiš Škerl M, Vlainić J, Sikirić P. Stable gastric pentadecapeptide BPC 157 in honeybee (Apis mellifera) therapy, to control Nosema ceranae invasions in apiary conditions. J Vet Pharmacol Ther 2018;41:614-621.

150. Sikiric P, Seiwerth S, Aralica G, et al. Therapy effect of antiulcer agents on new chronic cysteamine colon lesion in rat. J Physiol Paris 2001;95:283-288.

151. Sikiric P, Seiwerth S, Grabarevic Z, et al. Cysteamine-colon and cysteamine-duodenum lesions in rats. Attenuation by gastric pentadecapeptide BPC 157, cimetidine, ranitidine, atropine, omeprazole, sulphasalazine and methylprednisolone. J Physiol
Paris 2001;95:261-270.

152. Sikirić P, Mazul B, Seiwerth S, et al. Pentadecapeptide BPC 157 interactions with adrenergic and dopaminergic systems in mucosal protection in stress. Dig Dis Sci 1997;42:661-671.

153. Sikirić P, Seiwerth S, Grabarević Z, et al. Salutary and prophylactic effect of pentadecapeptide BPC 157 on acute pancreatitis and concomitant gastroduodenal lesions in rats. Dig Dis Sci 1996;41:1518-1526.

154. Sikiric P, Seiwerth S, Grabarevic Z, et al. Hepatoprotective effect of BPC 157, a 15-amino acid peptide, on liver lesions induced by either restraint stress or bile duct and hepatic artery ligation or $\mathrm{CCl} 4$ administration. A comparative study with dopamine agonists and somatostatin. Life Sci 1993;53:PL291-PL296.

155. Xue XC, Wu YJ, Gao MT, et al. Protective effects of pentadecapeptide BPC 157 on gastric ulcer in rats. World J Gastroenterol 2004;10:1032-1036. 